\title{
GAUSS-MANIN SYSTEMS, BRIESKORN LATTICES AND FROBENIUS STRUCTURES (II)
}

\author{
By Antoine Douai \& Claude Sabbah
}

Dedicated to Yuri Manin

\begin{abstract}
We give an explicit description of the canonical Frobenius structure attached (by the results of the first part of this article) to the polynomial $f\left(u_{0}, \ldots, u_{n}\right)=$ $w_{0} u_{0}+\cdots+w_{n} u_{n}$ restricted to the torus $U=\left\{\left(u_{0}, \ldots, u_{n}\right) \in \mathbb{C}^{n+1} \mid \prod_{i} u_{i}^{w_{i}}=1\right\}$, for any family of positive integers $w_{0}, \ldots, w_{n}$ such that $\operatorname{gcd}\left(w_{0}, \ldots, w_{n}\right)=1$.

RÉSUMÉ (Systèmes de Gauss-Manin, réseaux de Brieskorn et structures de Frobenius (II))

Nous donnons une description explicite de la structure de Frobenius associée (par les résultats de la première partie de cet article) au polynôme $f\left(u_{0}, \ldots, u_{n}\right)=w_{0} u_{0}+$ $\cdots+w_{n} u_{n}$ restreint au tore $U=\left\{\left(u_{0}, \ldots, u_{n}\right) \in \mathbb{C}^{n+1} \mid \prod_{i} u_{i}=1\right\}$ pour toute famille de poids $w_{0}, \ldots, w_{n}$ tels que $\operatorname{pgcd}\left(w_{0}, \ldots, w_{n}\right)=1$.
\end{abstract}

\section{Introduction}

1.a. This article explains a detailed example of the general result developed in the first part [3]. We were motivated by [1], where S. Barannikov describes a Frobenius structure attached to the Laurent polynomial $f\left(u_{0}, \ldots, u_{n}\right)=u_{0}+$ $\cdots+u_{n}$ restricted to the torus $U=\left\{\left(u_{0}, \ldots, u_{n}\right) \in \mathbb{C}^{n+1} \mid \prod_{i} u_{i}=1\right\}$, and

Antoine DouaI, UMR 6621 du CNRS, Laboratoire J.A. Dieudonné, Université de Nice, Parc Valrose, 06108 Nice cedex 2, France • E-mail : douai@math.unice.fr Claude SabBah, UMR 7640 du CNRS, Centre de Mathématiques, École polytechnique, F91128 Palaiseau cedex, France • E-mail : sabbah@math.polytechnique.fr Url : http://www.math.polytechnique.fr/cmat/sabbah/sabbah.html

2000 Mathematics Subject Classification. - 32S40, 32S30, 32G34, 32G20, 34Mxx.

Key words and phrases. - Gauss-Manin system, Brieskorn lattice, Frobenius manifold. 
shows that it is isomorphic to the Frobenius structure attached to the quantum cohomology of the projective space $\mathbb{P}^{n}(\mathbb{C})$ (as defined e.g., in [5]).

We will freely use the notation introduced in the first part [3]. A reference like "§ I.3.c" will mean [3, §3.c].

In the following, we fix an integer $n \geqslant 2$ and positive integers $w_{0}, \ldots, w_{n}$ such that $\operatorname{gcd}\left(w_{0}, \ldots, w_{n}\right)=1$. It will be convenient to assume that $w_{0} \leqslant \cdots \leqslant w_{n}$. We put

$$
\mu:=\sum_{i=0}^{n} w_{i} .
$$

We will analyze the Gauss-Manin system attached to the Laurent polynomial

$$
f\left(u_{1}, \ldots, u_{n}\right)=w_{0} u_{0}+w_{1} u_{1}+\cdots+w_{n} u_{n}
$$

restricted to the subtorus $U \subset\left(\mathbb{C}^{*}\right)^{n+1}$ defined by the equation

$$
u_{0}^{w_{0}} \cdots u_{n}^{w_{n}}=1 \text {. }
$$

The case $\mu=n+1$ (and all $w_{i}$ equal to 1 ) was considered in [1]. We will not need any explicit use of Hodge Theory, as all computation can be made "by hand". We will use the method of $\S$ I.3.c to obtain information concerning the Frobenius structure on any germ of universal deformation space of $f$. As we have seen in [3], the main point is to construct a primitive homogeneous element $\omega^{o}$, hence to analyze with some details the structure of the Gauss-Manin system and the Brieskorn lattice of $f$.

1.b. Fix a $\mathbb{Z}$-basis of $\left\{\sum_{i} w_{i} x_{i}=0\right\} \subset \mathbb{Z}^{n+1}$. It defines a $(n+1) \times n$ matrix $M$. Denote by $m_{0}, \ldots, m_{n}$ the lines of this matrix. We thus get a parametrization of $U$ by $\left(\mathbb{C}^{*}\right)^{n}$ by putting $u_{i}=v^{m_{i}}$ for $i=0, \ldots, n$ and $v=\left(v_{1}, \ldots, v_{n}\right)$. The vectors $m_{0}, \ldots, m_{n}$ are the vertices of a simplex $\Delta \subset \mathbb{Z}^{n}$, which is nothing but the Newton polyhedron of $f$ when expressed in the coordinates $v$. Notice that the determinant of the $n \times n$ matrix $\left(m_{0}, \ldots, \widehat{m_{i}}, \ldots, m_{n}\right)$ is $\pm w_{i}$.

LEMMA 1.2. - The Laurent polynomial $f$ is convenient and nondegenerate with respect to its Newton polyhedron.

Proof. - The nondegeneracy follows from the linear independence of any $n$ distinct vectors among $m_{0}, \ldots, m_{n}$. Clearly, 0 is contained in the interior of $\Delta$.

We know then that $f$ is M-tame $(c f . \S$ I.4) and we may therefore apply the results of $\S \mathrm{I} .2$ to $f$. An easy computation shows that $f$ has $\mu$ simple critical points, which are the $\zeta(1, \ldots, 1)$ with $\zeta^{\mu}=1$, and thus $\mu$ distinct critical values $\mu \zeta$. We hence have $\mu(f)=\mu$. 
1.c. Denote by $\mathcal{S}_{w}$ the disjoint union of the sets

$$
\left\{\ell \mu / w_{i} \mid \ell=0, \ldots, w_{i}-1\right\} \subset \mathbb{Q} .
$$

Hence $\# \mathcal{S}_{w}=\mu$. Number the elements of $\mathcal{S}_{w}$ from 0 to $\mu-1$ in an increasing way, with respect to the usual order on $\mathbb{Q}$. We therefore have $\mathcal{S}_{w}=$ $\left\{s_{w}(0), \ldots, s_{w}(\mu-1)\right\}$ with $s_{w}(k) \leqslant s_{w}(k+1)$. In particular, we have

$$
s_{w}(0)=\cdots=s_{w}(n)=0, \quad s_{w}(n+1)=\frac{\mu}{\max _{i} w_{i}}<n+1 .
$$

Moreover, using the involution $\ell \mapsto w_{i}-\ell$ for $\ell \geqslant 1$, one obtains, for $k \geqslant n+1$, the relation

$$
s_{w}(k)+s_{w}(\mu+n-k)=\mu .
$$

We consider the function $\sigma_{w}:\{0, \ldots, \mu-1\} \rightarrow \mathbb{Q}$ defined by

$$
\sigma_{w}(k)=k-s_{w}(k) \text {. }
$$

Hence $\sigma_{w}(k)=k$ for $k=0, \ldots, n$. That $s_{w}(\bullet)$ is increasing is equivalent to

$$
\forall k=0, \ldots, \mu-1, \quad \sigma_{w}(k+1) \leqslant \sigma_{w}(k)+1,
$$

where we use the convention $\sigma_{w}(\mu)=\sigma_{w}(0)=0$. We will prove:

THEOREM 1. - The polynomial $\prod_{k=0}^{\mu-1}\left(S+\sigma_{w}(k)\right)$ is equal to the spectral polynomial $\mathrm{SP}_{f}(S)$ attached to $f$ (cf. §I.2.e).

For instance, if we take the Laurent polynomial $f\left(u_{0}, \ldots, u_{n}\right)$ on the torus $\prod u_{i}=1$, i.e., $w_{0}=\cdots=w_{n}=1$, we get $\operatorname{SP}_{f}(S)=\prod_{k=0}^{n}(S+k)$.

Notice that the symmetry property (1.3) is a little bit more precise than the symmetry of the spectrum $(c f .[9])$, which would say that, for any $j \in$ $\{0, \ldots, n\}$,

$$
\#\left\{k \mid \sigma_{w}(k)=j\right\}=\#\left\{k \mid \sigma_{w}(k)=n-j\right\} .
$$

Indeed, for $k \in\{n+1, \ldots, \mu-1\},(1.3)$ means that $\sigma_{w}(k)+\sigma_{w}(\mu+n-k)=n$ and we clearly have $\sigma_{w}(k)+\sigma_{w}(n-k)=n$ for $k=0, \ldots, n$.

1.d. Consider now the two $\mu \times \mu$ matrices

$$
A_{\infty}=\operatorname{diag}\left(\sigma_{w}(0), \ldots, \sigma_{w}(\mu-1)\right), \quad A_{0}=\mu\left(\begin{array}{ccccc}
0 & & & & 1 \\
1 & 0 & & & 0 \\
0 & 1 & 0 & & 0 \\
\vdots & \ddots & \ddots & \ddots & \vdots \\
0 & \ldots & 0 & 1 & 0
\end{array}\right) .
$$

Notice that $A_{0}$ is semisimple with distinct eigenvalues $\mu \zeta$, where $\zeta$ is a $\mu$-th primitive root of 1 . In the canonical basis $\left(e_{0}, \ldots, e_{\mu-1}\right)$ of the space $\mathbb{C}^{\mu}$ on 
which these matrices act, consider the nondegenerate bilinear form $g$ defined by

$$
g\left(e_{k}, e_{\ell}\right)= \begin{cases}1 & \begin{array}{l}
\text { if } 0 \leqslant k \leqslant n \text { and } k+\ell=n, \\
\text { or if } n+1 \leqslant k \leqslant \mu-1 \text { and } k+\ell=\mu+n,
\end{array} \\
0 \quad \text { otherwise }\end{cases}
$$

with respect to which $A_{\infty}$ satisfies $A_{\infty}+{ }^{t} A_{\infty}=n \mathrm{Id}$. The data $\left(A_{0}, A_{\infty}, g, e_{0}\right)$ define (cf. [4, Main Theorem p.188], see also [5, §II.3] or [10, Th.VII.4.2]) a unique germ of semisimple Frobenius manifold at the point $\left(\mu, \mu \zeta, \ldots, \mu \zeta^{\mu-1}\right) \in$ $\mathbb{C}^{\mu}$.

The main result of this article is then:

TheOREM 2. - The canonical Frobenius structure on any germ of a universal unfolding of the Laurent polynomial $f\left(u_{0}, \ldots, u_{n}\right)=\sum_{i} w_{i} u_{i}$, as defined in [3], is isomorphic to the germ of universal semisimple Frobenius structure with initial data $\left(A_{0}, A_{\infty}, g, e_{0}\right)$ at the point $\left(\mu, \mu \zeta, \ldots, \mu \zeta^{\mu-1}\right) \in \mathbb{C}^{\mu}$.

REMARK. - It would be interesting to give an explicit description of the Gromov-Witten potential attached to this Frobenius structure.

\section{The rational numbers $\sigma_{w}(k)$}

Let us be now more precise on the definition of $s_{w}(k)$. Define inductively the sequence $(a(k), i(k)) \in \mathbb{N}^{n+1} \times\{0, \ldots, n\}$ by

$$
\begin{aligned}
a(0) & =(0, \ldots, 0), & i(0) & =0 \\
a(k+1) & =a(k)+\mathbf{1}_{i(k)}, & i(k+1) & =\min \left\{i \mid a(k+1)_{i} / w_{i}=\min _{j} a(k+1)_{j} / w_{j}\right\} .
\end{aligned}
$$

It is immediate that $|a(k)|:=\sum_{i=0}^{n} a(k)_{i}=k$ and that, for $k \leqslant n+1$, we have $a(k)_{i}=1$ if $i<k$ and $a(k)_{i}=0$ if $i \geqslant k+1$. In particular, $a(n+1)=(1, \ldots, 1)$.

Lemma 2.1. - The sequence $(a(k), i(k))$ satisfies the following properties:

(1) for all $k \in \mathbb{N}, \frac{a(k)_{i(k)}}{w_{i(k)}} \leqslant \frac{a(k+1)_{i(k+1)}}{w_{i(k+1)}} \leqslant \frac{a(k)_{i(k)}+1}{w_{i(k)}}$,

(2) $a(\mu)=\left(w_{0}, \ldots, w_{n}\right)$ and for all $k \in\{0, \ldots, \mu-1\}$, we have $a(k)_{i(k)} \leqslant$ $w_{i(k)}-1$,

(3) the map $\{0, \ldots, \mu-1\} \rightarrow \coprod_{i=0}^{n}\left\{0, \ldots, w_{i}-1\right\}$, defined by $k \mapsto$ $\left[i(k), a(k)_{i(k)}\right]$ is bijective.

(4) For $\ell \in \mathbb{N}$, we have $i(k+\ell \mu)=i(k)$ and $a(k+\ell \mu)_{i(k)}=\ell w_{i(k)}+a(k)_{i(k)}$.

We will then put $s_{w}(k):=\mu a(k)_{i(k)} / w_{i(k)}$. We have $s_{w}(k+\ell \mu)=\ell \mu+s_{w}(k)$ for $\ell \in \mathbb{N}$. 
Proof

(1) By induction on $k$. If $i(k+1)=i(k)$, the result is clear. Otherwise, we have $a(k+1)_{i(k+1)} / w_{i(k+1)}=a(k)_{i(k+1)} / w_{i(k+1)}$ and the first inequality follows from the definition of $i(k)$. Similarly, the second inequality is given by the definition of $i(k+1)$.

(2) Let us first remark the implication

$$
a(k)_{j} \leqslant w_{j} \forall j \text { and }\left\{j \mid a(k)_{j}<w_{j}\right\} \neq \varnothing \Longrightarrow a(k+1)_{j} \leqslant w_{j} \forall j .
$$

[Indeed, from the assumption we have $a(k)_{i(k)}<w_{i(k)}$, hence $a(k+1)_{i(k)}=$ $a(k)_{i(k)}+1 \leqslant w_{i(k)}$. For $j \neq i(k), a(k+1)_{j}=a(k)_{j} \leqslant w_{j}$.] Therefore, there exists $k_{0}$ such that $a\left(k_{0}\right)=\left(w_{0}, \ldots, w_{n}\right)$. Then $k_{0}=\left|a\left(k_{0}\right)\right|=\mu$. Moreover, by what we have just seen, we have $a(k)_{i(k)}<w_{i(k)}$ for $k<\mu$.

(3) The map does exist, after (2), is clearly injective, therefore bijective as the two sets have the same number of elements.

(4) We have $a(\mu)=\left(w_{0}, \ldots, w_{n}\right)$, so that $i(\mu)=0$, and we may apply the reasoning of (2) for $k=\mu, \ldots, 2 \mu-1$, etc.

REMARK 2.2. - In general, the numbers $s_{w}(k)$ are rational. These are integers (hence the spectrum of $f$ is integral) if and only if the following condition holds:

$$
\forall i, \quad w_{i} \mid \mu=w_{0}+\cdots+w_{n} .
$$

Consider the simplex $\Delta(w)$ in $\mathbb{R}^{n}$ obtained as the intersection of the hyperplane $\mathcal{H}=\left\{\sum_{i=0}^{n} w_{i} x_{i}=0\right\} \subset \mathbb{R}^{n+1}$ with the half spaces $x_{i} \geqslant-1$. Fix also the lattice $\mathcal{H}_{\mathbb{Z}}=H \cap \mathbb{Z}^{n+1}$. Then Condition (2.3) is equivalent to the condition that the vertices of $\Delta(w)$ are contained in the lattice $\mathcal{H}_{\mathbb{Z}}$. In other words, $\Delta(w)$ is a reflexive simplex in the sense of Batyrev [2]. For instance, if $n=3$, one finds the following possibilities for $w_{i}$ (up to a permutation):

\begin{tabular}{|r|r|r|r||r|}
\hline$w_{0}$ & $w_{1}$ & $w_{2}$ & $w_{3}$ & $\mu$ \\
\hline 1 & 1 & 1 & 1 & $\mathbf{4}$ \\
\hline 1 & 1 & 1 & 3 & $\mathbf{6}$ \\
\hline 1 & 1 & 2 & 2 & $\mathbf{6}$ \\
\hline 1 & 1 & 2 & 4 & $\mathbf{8}$ \\
\hline 1 & 2 & 2 & 5 & $\mathbf{1 0}$ \\
\hline 1 & 1 & 4 & 6 & $\mathbf{1 2}$ \\
\hline
\end{tabular}

\begin{tabular}{|r|r|r|r||r|}
\hline$w_{0}$ & $w_{1}$ & $w_{2}$ & $w_{3}$ & $\mu$ \\
\hline 1 & 2 & 3 & 6 & $\mathbf{1 2}$ \\
\hline 1 & 3 & 4 & 4 & $\mathbf{1 2}$ \\
\hline 1 & 2 & 6 & 9 & $\mathbf{1 8}$ \\
\hline 1 & 4 & 5 & 10 & $\mathbf{2 0}$ \\
\hline 1 & 3 & 8 & 12 & $\mathbf{2 4}$ \\
\hline 2 & 3 & 10 & 15 & $\mathbf{3 0}$ \\
\hline 1 & 6 & 14 & 21 & $\mathbf{4 2}$ \\
\hline
\end{tabular}


For $n=4$, here are some examples (maybe not complete):

\begin{tabular}{|r|r|r|r|r||r|}
\hline$w_{0}$ & $w_{1}$ & $w_{2}$ & $w_{3}$ & $w_{4}$ & $\mu$ \\
\hline 1 & 1 & 1 & 1 & 2 & $\mathbf{6}$ \\
\hline 1 & 1 & 2 & 2 & 2 & $\mathbf{8}$ \\
\hline 1 & 1 & 1 & 1 & 4 & $\mathbf{8}$ \\
\hline 1 & 1 & 1 & 3 & 3 & $\mathbf{9}$ \\
\hline 1 & 1 & 1 & 2 & 5 & $\mathbf{1 0}$ \\
\hline 2 & 2 & 2 & 3 & 3 & $\mathbf{1 2}$ \\
\hline 1 & 1 & 3 & 3 & 4 & $\mathbf{1 2}$ \\
\hline 1 & 1 & 2 & 2 & 6 & $\mathbf{1 2}$ \\
\hline 1 & 1 & 1 & 3 & 6 & $\mathbf{1 2}$ \\
\hline 1 & 1 & 3 & 5 & 5 & $\mathbf{1 5}$ \\
\hline
\end{tabular}

\begin{tabular}{|r|r|r|r|r||r|}
\hline$w_{0}$ & $w_{1}$ & $w_{2}$ & $w_{3}$ & $w_{4}$ & $\mu$ \\
\hline 1 & 1 & 2 & 4 & 8 & $\mathbf{1 6}$ \\
\hline 1 & 1 & 4 & 4 & 10 & $\mathbf{2 0}$ \\
\hline 1 & 1 & 4 & 6 & 12 & $\mathbf{2 4}$ \\
\hline 1 & 1 & 2 & 8 & 12 & $\mathbf{2 4}$ \\
\hline 1 & 1 & 3 & 10 & 15 & $\mathbf{3 0}$ \\
\hline 1 & 1 & 4 & 12 & 18 & $\mathbf{3 6}$ \\
\hline 1 & 1 & 8 & 10 & 20 & $\mathbf{4 0}$ \\
\hline 1 & 1 & 6 & 16 & 24 & $\mathbf{4 8}$ \\
\hline 1 & 1 & 8 & 20 & 30 & $\mathbf{6 0}$ \\
\hline 1 & 2 & 12 & 15 & 30 & $\mathbf{6 0}$ \\
\hline
\end{tabular}

\section{The Gauss-Manin system}

The Gauss-Manin system $G$ of the Laurent polynomial $f$ is a module over the ring $\mathbb{C}\left[\tau, \tau^{-1}\right]$. It is defined as in $\S$ I.2.c:

$$
G=\Omega^{n}(U)\left[\tau, \tau^{-1}\right] /(d-\tau d f \wedge) \Omega^{n-1}(U)\left[\tau, \tau^{-1}\right] .
$$

Put $\theta=\tau^{-1}$. The Briekorn lattice $G_{0}=\operatorname{image}\left(\Omega^{n}(U) \rightarrow G\right)$ is a free $\mathbb{C}[\theta]$ module of rank $\mu$ because, by Lemma $1.2, f$ is convenient and nondegenerate (loc. cit.). We will consider the increasing filtration $G_{p}=\tau^{-p} G_{0}(p \in \mathbb{Z})$. Let $\omega_{0}$ be the $n$-form on $U$ defined by

$$
\omega_{0}=\left.\frac{\frac{d u_{0}}{u_{0}} \wedge \cdots \wedge \frac{d u_{n}}{u_{n}}}{d\left(\prod_{i} u_{i}^{w_{i}}\right)}\right|_{\prod_{i} u_{i}^{w_{i}}=1} .
$$

Let $v \mapsto u=v^{m}$ be a parametrization of $U$ as in $\S 1$.b. The form $\omega_{0}$ can be written as $\omega_{0}= \pm \frac{d v_{1}}{v_{1}} \wedge \cdots \wedge \frac{d v_{n}}{v_{n}}$. The Gauss-Manin system $G$ is then identified with the $\mathbb{C}\left[\tau, \tau^{-1}\right]$-module (putting $v=\left(v_{1}, \ldots, v_{n}\right)$ )

$\mathbb{C}\left[v, v^{-1}, \tau, \tau^{-1}\right] /\left\{v_{j} \partial_{v_{j}}\left(\varphi_{j}\right)-\tau\left(v_{j} \partial_{v_{j}} f\right) \varphi_{j} \mid \varphi_{j} \in \mathbb{C}\left[v, v^{-1}, \tau, \tau^{-1}\right], j=1, \ldots, n\right\}$.

It comes equipped with an action of $\partial_{\tau}$ : if $\psi \in \mathbb{C}\left[v, v^{-1}\right]$, let $[\psi]$ denote its class in $G$; then $\partial_{\tau}[\psi]=[-f \psi]$ (this does not depend on the representative of the class). Using the coordinate $\theta$, we have $\theta^{2} \partial_{\theta}[\psi]=[f \psi]$; this action is extended in the usual way to Laurent polynomials in $\tau$ with coefficients like $[\psi]$. 
It is convenient to use the coordinates $u=\left(u_{0}, \ldots, u_{n}\right)$. Then the previous quotient is written as

$$
\mathbb{C}\left[u, u^{-1}, \tau, \tau^{-1}\right] /\left(I_{w}+\mathbb{C}\left[u, u^{-1}, \tau, \tau^{-1}\right](g(u)-1)\right),
$$

where we have put $g(u)=\prod_{i} u_{i}^{w_{i}}$ and $I_{w}$ is the $\mathbb{C}\left[\tau, \tau^{-1}\right]$-submodule of $\mathbb{C}\left[u, u^{-1}, \tau, \tau^{-1}\right]$ consisting of the expressions

(3.1) $\sum_{i=0}^{n} m_{j i}\left(u_{i} \frac{\partial}{\partial u_{i}}-\tau w_{i} u_{i}\right) \varphi_{j}$, with $\varphi_{j} \in \mathbb{C}\left[u, u^{-1}, \tau, \tau^{-1}\right], \quad(j=1, \ldots, n)$.

Consider the sequence $(a(k), i(k))$ of Lemma 2.1 , and for each $k=0, \ldots, \mu$, put

$$
\omega_{k}=u^{a(k)} \omega_{0} \in G_{0}
$$

Notice that $\omega_{\mu}=\omega_{0}$ and, using (3.5) below, that $f \omega_{0}=\mu \omega_{1}$.

Proposition 3.2. - The classes of $\omega_{0}, \omega_{1}, \ldots, \omega_{\mu-1}$ form a $\mathbb{C}[\theta]$-basis $\boldsymbol{\omega}$ of $G_{0}$. Moreover, they satisfy the equation

$$
-\frac{1}{\mu}\left(\tau \partial_{\tau}+\sigma_{w}(k)\right) \omega_{k}=\tau \omega_{k+1} \quad(k=0, \ldots, \mu-1),
$$

and we have Bernstein's relation in $G$ :

$$
\prod_{k=0}^{\mu-1}\left[-\frac{1}{\mu}\left(\tau \partial_{\tau}-s_{w}(k)\right)\right] \cdot \omega_{0}=\tau^{\mu} \omega_{0} .
$$

The $V$-order of $\omega_{k}$ is equal to $\sigma_{w}(k)$ and $\boldsymbol{\omega}$ induces a $\mathbb{C}$-basis of $\oplus_{\alpha} \operatorname{gr}_{\alpha}^{V}\left(G_{0} / G_{-1}\right)$.

From Theorem I.4.5, Lemma I.4.3(3), and the symmetry (1.3), we get

$$
\text { for } k=0, \ldots, \mu-1, \quad 0 \leqslant \sigma_{w}(k) \leqslant n \text { and }\left\{\begin{array}{l}
\sigma_{w}(k)=0 \Rightarrow k=0 \\
\sigma_{w}(k)=n \Rightarrow k=n
\end{array}\right.
$$

This implies that, for any $\alpha \in] 0, n$ [, the length of a maximal subsequence $\alpha, \alpha+1, \ldots, \alpha+\ell$ of $\sigma_{w}(\bullet)$ is $\leqslant n$, and even $\leqslant n-1$ if $\alpha$ is an integer. In other words:

Corollary 3.4. - The length of any maximal nonzero integral (resp. nonintegral) constant subsequence of $s_{w}(\bullet)$ is $\leqslant n-1$ (resp. $\left.\leqslant n\right)$.

The proposition also gives a Birkhoff normal form for $G_{0}$ :

$$
\theta^{2} \partial_{\theta} \boldsymbol{\omega}=\boldsymbol{\omega} A_{0}+\theta \boldsymbol{\omega} A_{\infty}
$$

with $A_{0}, A_{\infty}$ as in (1.6). The matrix $A_{0}$ is nothing but the matrix of multiplication by $f$ on $G_{0} / \theta G_{0}$ in the basis induced by $\boldsymbol{\omega}$. Its eigenvalues are the critical values of $f$, as expected. In the case where $\mu=n+1$ (and all $w_{i}$ equal to 1 ), we find that $A_{\infty}=\operatorname{diag}(0,1, \ldots, n)$ and $A_{0}$ is as in (1.6) with size $\mu=n+1$. 
Proof of Proposition 3.2. - It will be convenient to select some coordinate, say $u_{0}$. Multiplying (3.1) (applied to $\varphi_{1}=\cdots=\varphi_{n}=\varphi$ ) on the left by the inverse matrix of the matrix formed by the columns of $m_{1}, \ldots, m_{n}$, one finds that, for any $\varphi \in \mathbb{C}\left[u, u^{-1}, \tau, \tau^{-1}\right]$, we have in $G$

$$
\forall i=1, \ldots, n \quad\left(\frac{1}{w_{i}} u_{i} \partial_{u_{i}}-\frac{1}{w_{0}} u_{0} \partial_{u_{0}}\right) \varphi \omega_{0}=\tau\left(u_{i}-u_{0}\right) \varphi \omega_{0} .
$$

Applying this to any monomial $\varphi=u^{a}$ and summing these equalities, we get the following relation for $j=0$, hence for any $j=0, \ldots, n$ by a similar argument:

$$
-\frac{1}{\mu}\left(\tau \partial_{\tau}+L_{j}(a)\right) u^{a} \omega_{0}=\tau u^{a+\mathbf{1}_{j}} \omega_{0},
$$

where we put $L_{j}(a)=\sum_{i=0}^{n} a_{i}-\mu a_{j} / w_{j}$. This is nothing but (I.4.12) in the present situation. Apply this for $a=a(k)$ and $j=i(k)(k=0, \ldots, \mu-1)$ to get the first relation in the lemma (remark that $\left.L_{i(k)}(a(k))=\sigma_{w}(k)\right)$. Bernstein's relation for $\omega_{0}$ is then clear. Remark also that $\omega_{k}$ is given by

$$
\omega_{k}=\tau^{-k} \prod_{j=0}^{k-1}\left[-\frac{1}{\mu}\left(\tau \partial_{\tau}-s_{w}(j)\right)\right] \cdot \omega_{0} .
$$

It is not difficult to derive from Bernstein's relation for $\omega_{0}$ a Bernstein relation for each $\omega_{k}$ and conclude that $\omega_{k}$ has $V$-order $\leqslant \sigma_{w}(k)$. [Notice also that, as $\sigma_{w}(k)=L_{i(k)}(a(k))=\max _{j} L_{j}(a(k))$, the order of $\omega_{k}$ with respect to the Newton filtration is $\leqslant \sigma_{w}(k)$; this is compatible with Theorem I.4.5.]

Let us now show that $\omega_{0}, \ldots, \omega_{\mu-1}$ generate $G_{0}$ as a $\mathbb{C}[\theta]$-module. Notice that Bernstein's relation for $\omega_{0}$ implies that $\partial_{\tau}^{\mu} \omega_{0} \in \mathbb{C}[\theta]\left\langle\omega_{0}, \ldots, \partial_{\tau}^{\mu-1} \omega_{0}\right\rangle=$ $\mathbb{C}[\theta]\left\langle\omega_{0}, \ldots, \omega_{\mu-1}\right\rangle$, and this also holds for $\partial_{\tau}^{\ell} \omega_{0}$ for $\ell \geqslant \mu$. It is therefore enough to show that $\left(f^{\ell} \omega_{0}\right)_{\ell \geqslant 0}$ generate $G_{0}$ over $\mathbb{C}[\theta]$. Write (3.5) as

$$
u^{a+\mathbf{1}_{i}} \omega_{0}=\left[u^{a} u_{0}+\left(\frac{a_{i}}{w_{i}}-\frac{a_{0}}{w_{0}}\right) \theta u^{a}\right] \omega_{0} .
$$

The Brieskorn lattice $G_{0}$ is generated over $\mathbb{C}[\theta]$ by the $u_{0}^{\ell} \omega_{0}$ with $\ell \in \mathbb{N}$ : indeed, it is generated by the $u^{a} \omega_{0}$; then,

- if $a_{i} \geqslant 1$ for some $i \geqslant 1$, one decreases $a_{i}$ to 0 with (3.7);

- if $a_{i} \leqslant-1$ for some $i \geqslant 1$, one iterates (3.7) $w_{0}$ times and use the relation $u^{w} \omega_{0}=\omega_{0}$ to express $u^{b} \omega_{0}$ (any $b$ ) as a sum (with constant coefficients) of terms $\theta^{k} u_{0}^{\ell} u^{b+w^{\prime}} \omega_{0}$ and of $u^{b+w^{\prime}+\mathbf{1}_{i}}$, with $k, \ell \geqslant 0$ and $w^{\prime}=\left(0, w_{1}, \ldots, w_{n}\right)$; hence if $b_{i}<0$, there exists $r$ such that $b_{i}+r w_{i} \geqslant 0$ and one iterates $r$ times the previous process to write $u^{b} \omega_{0}$ with terms $\theta^{k} u^{a}$, with $a_{i} \geqslant 1$, to reduce to the previous case; 
- notice that, in both previous processes, we never decrease the degree in $u_{0}$; now, we are reduced to considering $u_{0}^{\ell} \omega_{0}$ with $\ell<0$; use once more the relation $u^{k w} u^{b} \omega_{0}=u^{b} \omega_{0}$ (for any $k \geqslant 0$, any $b$ ) to replace $u_{0}^{\ell}$ with $u^{a}$ with $a_{0}, \ldots, a_{n} \geqslant 0$ and apply the first case.

A similar argument gives the result for the family $\left(f^{\ell} \omega_{0}\right)_{\ell \geqslant 0}$. As $G_{0}$ is $\mathbb{C}[\theta]$-free (cf. Remark I.4.8 and $\S$ I.2.c), we conclude that $\boldsymbol{\omega}$ is a $\mathbb{C}[\theta]$-basis of $G_{0}$. [Instead of using Remark I.4.8, one can directly conclude here that $G_{0}$ is $\mathbb{C}[\theta]$-free of rank $\mu$ by showing first that $\boldsymbol{\omega}$ generates $G$ as a $\mathbb{C}\left[\tau, \tau^{-1}\right]$-module. $]$

Remark also that $\left(\omega_{0}, \ldots, f^{\mu-1} \omega_{0}\right)$ is another basis, but the differential equation does not take Birkhoff normal form in such a basis.

We will now determine the $V$-filtration. Put $\omega_{k}^{\prime}=\tau^{\left[\sigma_{w}(k)\right]} \omega_{k}$. Then $\boldsymbol{\omega}^{\prime}$ is another $\mathbb{C}\left[\tau, \tau^{-1}\right]$-basis of $G$. The $V$-order of $\omega_{k}^{\prime}$ is $\leqslant \sigma_{w}(k)-\left[\sigma_{w}(k)\right]<1$. For $\alpha \in[0,1[$, put

$$
\begin{aligned}
U_{\alpha} G & =\mathbb{C}[\tau]\left\langle\omega_{k}^{\prime} \mid \sigma_{w}(k)-\left[\sigma_{w}(k)\right] \leqslant \alpha\right\rangle+\tau \mathbb{C}[\tau]\left\langle\omega_{k}^{\prime} \mid \sigma_{w}(k)-\left[\sigma_{w}(k)\right]>\alpha\right\rangle \\
U_{<\alpha} G & =\mathbb{C}[\tau]\left\langle\omega_{k}^{\prime} \mid \sigma_{w}(k)-\left[\sigma_{w}(k)\right]<\alpha\right\rangle+\tau \mathbb{C}[\tau]\left\langle\omega_{k}^{\prime} \mid \sigma_{w}(k)-\left[\sigma_{w}(k)\right] \geqslant \alpha\right\rangle,
\end{aligned}
$$

and $U_{\alpha+p} G=\tau^{p} U_{\alpha} G$ (resp. $\left.U_{<\alpha+p} G=\tau^{p} U_{<\alpha} G\right)$ for any $p \in \mathbb{Z}$. We then have

$$
U_{\alpha} G=\mathbb{C}\left\langle\omega_{k}^{\prime} \mid \sigma_{w}(k)-\left[\sigma_{w}(k)\right]=\alpha\right\rangle+U_{<\alpha} G .
$$

Notice that, according to the formula for $\omega_{k}$, the elements $\omega_{k}^{\prime}$ satisfy

$$
\begin{aligned}
-\frac{1}{\mu}\left(\tau \partial_{\tau}+\sigma_{w}(k)-\left[\sigma_{w}(k)\right]\right) \omega_{k}^{\prime} & =\tau^{\left[\sigma_{w}(k)\right]+1-\left[\sigma_{w}(k+1)\right]} \omega_{k+1}^{\prime} \\
& =\tau^{\left\lceil s_{w}(k+1)\right\rceil-\left\lceil s_{w}(k)\right\rceil} \omega_{k+1}^{\prime},
\end{aligned}
$$

with $\lceil s\rceil:=-[-s]$. Recall that the sequence $\left(s_{w}(k)\right)$, hence the sequence $\left(\left\lceil s_{w}(k)\right\rceil\right)$, is increasing. If $\left\lceil s_{w}(k+1)\right\rceil>\left\lceil s_{w}(k)\right\rceil$, then

$$
\left(\tau \partial_{\tau}+\sigma_{w}(k)-\left[\sigma_{w}(k)\right]\right) \omega_{k}^{\prime} \in U_{<0} G .
$$

Otherwise, we have

$$
\left\lceil s_{w}(k)\right\rceil-s_{w}(k) \geqslant\left\lceil s_{w}(k+1)\right\rceil-s_{w}(k+1),
$$

i.e.,

$$
\sigma_{w}(k)-\left[\sigma_{w}(k)\right] \geqslant \sigma_{w}(k+1)-\left[\sigma_{w}(k+1)\right],
$$

and we conclude that $U_{\alpha} G$ is stable under $\tau \partial_{\tau}$ and that $\tau \partial_{\tau}+\alpha$ is nilpotent on $\operatorname{gr}_{\alpha}^{U} G$. The filtration $U_{\bullet} G$ satisfies then the characterizing properties of $V_{\bullet} G$, hence is equal to it. 
We may now compute $G_{p} \cap V_{\alpha}$ for $p \in \mathbb{Z}$ and $\alpha \in[0,1[$. Any element of $G_{p} \cap V_{\alpha}$ decomposes uniquely as $\sum_{k=0}^{\mu-1} p_{k}(\tau) \omega_{k}^{\prime}$, with

$$
p_{k}(\tau) \in \begin{cases}\tau^{p-\left[\sigma_{w}(k)\right]} \mathbb{C}\left[\tau^{-1}\right] \cap \mathbb{C}[\tau] & \text { if } \sigma_{w}(k)-\left[\sigma_{w}(k)\right] \leqslant \alpha, \\ \tau^{p-\left[\sigma_{w}(k)\right]} \mathbb{C}\left[\tau^{-1}\right] \cap \tau \mathbb{C}[\tau] & \text { if } \sigma_{w}(k)-\left[\sigma_{w}(k)\right]>\alpha\end{cases}
$$

It follows that

$$
G_{p} \cap V_{\alpha}=\sum_{k \mid \sigma_{w}(k)=\alpha+p} \mathbb{C} \cdot \omega_{k}^{\prime}+G_{p} \cap V_{<\alpha}+G_{p-1} \cap V_{\alpha},
$$

and therefore $\operatorname{gr}_{p}^{G} \operatorname{gr}_{\alpha}^{V} G$ is generated by the classes of $\omega_{k}^{\prime}$ with $\sigma_{w}(k)=\alpha+p$. These classes form a basis of $\operatorname{gr}_{p}^{G} \operatorname{gr}_{\alpha}^{V} G$, as $\operatorname{dim} \oplus_{p} \oplus_{\alpha \in[0,1[} \operatorname{gr}_{p}^{G} \operatorname{gr}_{\alpha}^{V} G=\mu$. This gives the last statement of the proposition.

For $\alpha \in\left[0,1\left[\right.\right.$, let $\omega_{k}^{\prime}$ be such that $\left\lceil s_{w}(k)\right\rceil-s_{w}(k)=\alpha$ and denote by $\left[\omega_{k}^{\prime}\right]$ the class of $\omega_{k}^{\prime}$ in $H_{\alpha}:=\operatorname{gr}_{\alpha}^{V} G$. After (1.5) we have:

$$
-\frac{1}{\mu}\left(\tau \partial_{\tau}+\alpha\right)\left[\omega_{k}^{\prime}\right]= \begin{cases}0 & \text { if } s_{w}(k+1)>s_{w}(k), \\ {\left[\omega_{k+1}^{\prime}\right]} & \text { if } s_{w}(k+1)=s_{w}(k) .\end{cases}
$$

It follows that the primitive elements relative to the nilpotent operator induced by $(-1 / \mu)\left(\tau \partial_{\tau}+\alpha\right)$ on $H_{\alpha}$ are the elements $\left[\omega_{k}^{\prime}\right]$ such that

$$
k \geqslant n+1, \quad\left\lceil s_{w}(k)\right\rceil-s_{w}(k)=\alpha \quad \text { and } \quad s_{w}(k-1)<s_{w}(k)
$$

and, if moreover $\alpha=0$, the element $\left[\omega_{0}^{\prime}\right]=\left[\omega_{0}\right]$.

Therefore, the Jordan blocks of $(-1 / \mu)\left(\tau \partial_{\tau}+\alpha\right)$ on $H_{\alpha}$ are in one-to-one correspondence with the maximal constant sequences in $\mathcal{S}_{w}$, and the corresponding sizes are the same. All Jordan blocks, except that of $\left[\omega_{0}\right]$ if $\alpha=0$, have thus size $\leqslant n$, and even $\leqslant n-1$ if $\alpha$ is an integer (cf. Cor. 3.4). Recall also (cf. [8,9]) that $H:=\oplus_{\alpha \in[0,1[} H_{\alpha}$ may be identified with the relative cohomology space $H^{n}\left(U, f^{-1}(t)\right)$ for $|t| \gg 0$, that $H_{\alpha}$ correspond to the generalized eigenspace of the monodromy corresponding to the eigenvalue exp $2 i \pi \alpha$, and that the nilpotent part of the monodromy operator $T$ is equal to $\exp 2 i \pi N$ with $N:=-\left(\tau \partial_{\tau}+\alpha\right)$.

EXAMPle 3.11. - Take $n=4$ and $w_{0}=1, w_{1}=2, w_{2}=12, w_{3}=15$ and $w_{4}=30$, so that $\mu=60$. Then the only possible $\alpha$ is 0 and $N$ has one Jordan block of size 5,3 blocks of size 3,13 blocks of size 2 and 20 blocks of size 1 . On the other hand, if $\mu=n+1$ (and all $w_{i}$ equal to 1 ), the only possible $\alpha$ is 0 and $N$ has only one Jordan block (of size $n+1$ ). 


\section{Poincaré duality and higher residue pairings}

Consider on $\mathbb{C}\left[\tau, \tau^{-1}\right]$ the ring involution induced by $\tau \mapsto-\tau$. We will set $\overline{p(\tau)}:=p(-\tau)$ (there is no complex conjugation involved here). Given a $\mathbb{C}\left[\tau, \tau^{-1}\right]$-module $G$, we denote by $\bar{G}$ the $\mathbb{C}$-vector space $G$ equipped with the new module structure $p(\tau) \cdot g=p(-\tau) g$. For convenience, we denote by $\bar{g}$ the elements of $\bar{G}$. The $\mathbb{C}\left[\tau, \tau^{-1}\right]$-structure of $\bar{G}$ is therefore given by the rule: $\overline{p(\tau)} \bar{g}=\overline{p(\tau) g}$.

If $G$ is moreover equipped with a connection, i.e., with a compatible action of $\partial_{\tau}$, then so is $\bar{G}$ and we have $\partial_{\tau} \bar{g}:=\overline{-\partial_{\tau} g}$. Notice that $\tau \partial_{\tau} \bar{g}=\overline{\tau \partial_{\tau} g}$.

Duality for $\mathcal{D}$-modules gives (cf. [9]) the existence of a nondegenerate $\mathbb{C}\left[\tau, \tau^{-1}\right]$-bilinear pairing

$$
S: G \underset{\mathbb{C}\left[\tau, \tau^{-1}\right]}{\otimes} \bar{G} \longrightarrow \mathbb{C}\left[\tau, \tau^{-1}\right]
$$

satisfying the following properties:

(1) $\frac{d S\left(g^{\prime}, \overline{g^{\prime \prime}}\right)}{d \tau}=S\left(\partial_{\tau} g^{\prime}, \overline{g^{\prime \prime}}\right)+S\left(g^{\prime}, \partial_{\tau} \overline{g^{\prime \prime}}\right)=S\left(\partial_{\tau} g^{\prime}, \overline{g^{\prime \prime}}\right)-S\left(g^{\prime}, \overline{\partial_{\tau} g^{\prime \prime}}\right)$,

(equivalently, $\tau \partial_{\tau} S\left(g^{\prime}, \overline{g^{\prime \prime}}\right)=S\left(\tau \partial_{\tau} g^{\prime}, \overline{g^{\prime \prime}}\right)+S\left(g^{\prime}, \overline{\tau \partial_{\tau} g^{\prime \prime}}\right)$ ),

(2) $S$ sends $V_{0} \otimes \overline{V_{<1}}$ in $\mathbb{C}[\tau]$,

(3) $S$ sends $G_{0} \otimes \overline{G_{0}}$ in $\theta^{n} \mathbb{C}[\theta]=\tau^{-n} \mathbb{C}\left[\tau^{-1}\right]$,

(4) $S\left(g^{\prime \prime}, \overline{g^{\prime}}\right)=(-1)^{n} \overline{S\left(g^{\prime}, \overline{g^{\prime \prime}}\right)}$ (this reflects the $(-1)^{n}$-symmetry of the Poincaré duality on $U)$.

Notice that (1) means that $S$ is a horizontal section of the $\mathbb{C}\left[\tau, \tau^{-1}\right]$ module $\operatorname{Hom}_{\mathbb{C}\left[\tau, \tau^{-1}\right]}\left(G \otimes \bar{G}, \mathbb{C}\left[\tau, \tau^{-1}\right]\right)$ equipped with its natural connection, or also that $S$ is a $\mathbb{C}[\tau]\left\langle\partial_{\tau}\right\rangle$-linear morphism $\bar{G} \rightarrow G^{*}$, if one endows $G^{*}=\operatorname{Hom}_{\mathbb{C}\left[\tau, \tau^{-1}\right]}\left(G, \mathbb{C}\left[\tau, \tau^{-1}\right]\right)$ with its natural connection. Therefore, (2) follows from (1) because any $\mathbb{C}[\tau]\left\langle\partial_{\tau}\right\rangle$-linear morphism is strict with respect to the Malgrange-Kashiwara filtrations $V$ and we have

$$
V_{\beta}\left(G^{*}\right)=\operatorname{Hom}_{\mathbb{C}[\tau]}\left(V_{<-\beta+1} G, \mathbb{C}[\tau]\right)
$$

(cf. [9]).

In the case of singularities, this corresponds to the "higher residue pairings" of K. Saito [11]. The link with Poincaré duality is explained in [12].

For our Laurent polynomial $f$, we will recover in an elementary way the existence of such a pairing $S$ satisfying the previous properties. More precisely, we have: 
Lemma 4.1. - There exists a unique (up to a nonzero constant) nondegenerate pairing $S$ satisfying Properties (1), (2), (3). It is given by the formula:

$$
S\left(\omega_{k}, \bar{\omega}_{\ell}\right)= \begin{cases}S\left(\omega_{0}, \overline{\omega_{n}}\right) & \left\{\begin{array}{l}
\text { if } 0 \leqslant k \leqslant n \text { and } k+\ell=n, \\
\text { or if } n+1 \leqslant k \leqslant \mu-1 \text { and } k+\ell=\mu+n,
\end{array}\right. \\
0 & \text { otherwise. }\end{cases}
$$

Moreover, for any $k, \ell, S\left(\omega_{k}, \overline{\omega_{\ell}}\right)$ belongs to $\mathbb{C} \tau^{-n}$ and $S$ satisfies (4).

Proof. - Assume that a pairing $S$ satisfying (1), (2), (3) exists. For $k, \ell=$ $0, \ldots, \mu-1$, we have $S\left(\omega_{k}, \bar{\omega}_{\ell}\right) \in \tau^{-n} \mathbb{C}\left[\tau^{-1}\right]$ by $(3)$ and $S\left(\omega_{0}, \overline{\omega_{\ell}}\right) \in \tau^{-\left[\sigma_{w}(\ell)\right]} \mathbb{C}[\tau]$ by $(2)$. Therefore, $S\left(\omega_{0}, \overline{\omega_{\ell}}\right) \neq 0$ implies $\left[\sigma_{w}(\ell)\right] \geqslant n$, and if $\left[\sigma_{w}(\ell)\right]=n$, we have $S\left(\omega_{0}, \overline{\omega_{\ell}}\right) \in \mathbb{C} \tau^{-n}$. But we know by (3.3) that

$$
\left[\sigma_{w}(\ell)\right] \begin{cases}<n & \text { if } \ell \neq n, \\ =n & \text { if } \ell=n .\end{cases}
$$

Therefore, $S\left(\omega_{0}, \overline{\omega_{\ell}}\right)=0$ if $\ell \neq n$ and $S\left(\omega_{0}, \overline{\omega_{n}}\right) \in \mathbb{C} \tau^{-n}$.

Notice also that we have by (1) and Proposition 3.2:

$$
\begin{aligned}
& -\frac{1}{\mu}\left(\tau \partial_{\tau}+n\right) S\left(\omega_{k}, \overline{\omega_{\ell}}\right) \\
& \quad=\tau\left[S\left(\omega_{k+1}, \overline{\omega_{\ell}}\right)-S\left(\omega_{k}, \overline{\omega_{\ell+1}}\right)\right]+\frac{\sigma_{w}(k)+\sigma_{w}(\ell)-n}{\mu} S\left(\omega_{k}, \overline{\omega_{\ell}}\right),
\end{aligned}
$$

if we put as above $\omega_{\mu}=\omega_{0}$.

Argue now by induction for $k<n$ : as $S\left(\omega_{k}, \overline{\omega_{\ell}}\right) \in \mathbb{C} \tau^{-n}$, the LHS in (4.2) vanishes. This shows that $S\left(\omega_{k+1}, \overline{\omega_{\ell}}\right)=0$ if $\ell \neq n-k, n-1-k$. Moreover, if $\ell=n-k$, we have $\sigma_{w}(k)+\sigma_{w}(\ell)-n=0$, hence $S\left(\omega_{k+1}, \overline{\omega_{n-k}}\right)=0$. Last, we have $S\left(\omega_{k+1}, \overline{\omega_{n-1-k}}\right)=S\left(\omega_{k}, \overline{\omega_{n-k}}\right)$.

Argue similarly for $k \geqslant n+1$.

Notice that, if $A_{\infty}^{*}$ denotes the adjoint of $A_{\infty}$ with respect to $S$, then $A_{\infty}+$ $A_{\infty}^{*}=n \mathrm{Id}$, i.e., $A_{\infty}-(n / 2)$ Id is skewsymmetric with respect to $S$.

\section{M. Saito's solution to the Birkhoff problem}

One step in constructing the Frobenius structure associated to $f$ consists in solving Birkhoff's problem for the Brieskorn lattice $G_{0}$ in the Gauss-Manin system $G$, that is, in finding a $\mathbb{C}[\tau]$-lattice $E$ of $G$, which glues with $G_{0}$ to a trivial vector bundle on $\mathbb{P}^{1}$. Recall that there is a one-to-one correspondence between such lattices $E$ which are logarithmic, and decreasing filtrations $\oplus_{\alpha \in[0,1[} H_{\alpha}^{\bullet}$ 
of $H=\oplus_{\alpha \in[0,1[} H_{\alpha}$ which are stable under $N$ and which are opposite to the filtration

$$
G_{p}(H):=\underset{\alpha \in[0,1[}{\oplus}\left(G_{p} \cap V_{\alpha}\right) /\left(G_{p} \cap V_{<\alpha}\right)=\mathbb{C}\left\langle\left[\omega_{k}^{\prime}\right] \mid\left[\sigma_{w}(k)\right] \leqslant p\right\rangle \text { after (3.9). }
$$

This is analogous to [12, Th. 3.6] and follows for instance from [10, Th.IV.5.9 and Th. III.1.1].

In [12, Lemma 2.8], M. Saito defines a canonical decreasing filtration $H_{\text {Saito }}$

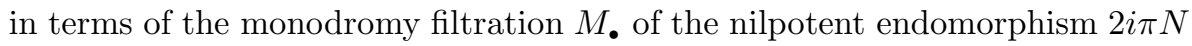
of $H$ and of the filtration conjugate to $G_{p}(H)$, the conjugation being taken with respect to the real structure on $H$ coming from the identification with $H^{n}\left(U, f^{-1}(t)\right)$. This defines therefore a canonical solution to Birkhoff's problem for $G_{0}$.

Consider now the decreasing filtration $H^{\bullet}$ of $H$ explicitly defined by

$$
H^{p}=\mathbb{C}\left\langle\left[\omega_{k}^{\prime}\right] \mid\left[\sigma_{w}(k)\right] \geqslant p\right\rangle,
$$

where [] denotes the class in $H=\oplus_{\alpha \in[0,1[} V_{\alpha} G / V_{<\alpha} G$. Then $H^{\bullet}$ is opposite to $G$ • $(H)$. It satisfies

$$
H^{0}=H, H^{n+1}=0, \quad N H^{p} \subset H^{p+1}
$$

and, for $k=0, \ldots, \mu-1$ and $\alpha \in[0,1[$,

$$
\left(H_{\alpha}^{p}\right)^{\perp}= \begin{cases}H_{1-\alpha}^{n-p} & \text { if } \alpha \neq 0, \\ H_{0}^{n+1-p} & \text { if } \alpha=0,\end{cases}
$$

where ${ }^{\perp}$ means taking the orthogonal with respect to the symmetric bilinear form $g$ on $H$ induced by $S$. If $\mu=n+1$ (and all $w_{i}$ equal to 1), then $H^{p}=$ $M_{n-2 p}$ (this implies that the mixed Hodge structure on $H$ is "Hodge-Tate").

Proposition 5.2. - The filtration $H^{\bullet}$ is equal to the opposite filtration $H_{\text {Saito }}^{\bullet}$. The associated logarithmic lattice is $E:=\mathbb{C}[\tau]\left\langle\omega_{0}, \ldots, \omega_{\mu-1}\right\rangle$.

Proof. - Let us begin with the second statement. The lattice $E$ introduced in the proposition is logarithmic, by Proposition 3.2. A computation analogous to that of $G_{p} \cap V_{\alpha}$ shows that the filtration $\tau^{p} E \cap V_{\alpha} / V_{<\alpha}$ of $H_{\alpha}$ is equal to $H_{\alpha}^{p}$. Therefore, $E$ is the logarithmic lattice corresponding to $H^{\bullet}$ by the correspondence recalled above.

For the first statement, put $F^{\bullet}(H)=G_{n-\bullet}(H)$. This is a decreasing filtration. Consider also the increasing filtration

$$
W_{\bullet}\left(H_{\alpha}\right)= \begin{cases}M_{\bullet-n-1}\left(H_{\alpha}\right) & \text { if } \alpha \neq 0 \\ M_{\bullet-n}\left(H_{0}\right) & \text { if } \alpha=0\end{cases}
$$


where $M_{\bullet}(H)$ denotes the monodromy filtration of the nilpotent endomorphism $2 i \pi N$ on $H$. Recall that $W \cdot(H)$ is defined over $\mathbb{R}$ (even over $\mathbb{Q}$ ) as $2 i \pi N$ is so. Then the opposite filtration given by M. Saito is

$$
H^{\prime \bullet}=\sum_{q} \bar{F}^{q} \cap W_{n+q-\bullet}(H)
$$

where $\bar{E}$ denotes the conjugate of the subspace $E$ of $H$ with respect to the complex conjugation coming from the identification

$$
H \stackrel{\sim}{\longrightarrow} H^{n}\left(U, f^{-1}(t), \mathbb{C}\right)=\mathbb{C} \otimes_{\mathbb{R}} H^{n}\left(U, f^{-1}(t), \mathbb{R}\right) .
$$

We therefore need to give a description of the conjugation in term of the basis $\left[\omega_{k}^{\prime}\right]$.

Let $k_{0}$ be such that $\left[\omega_{k_{0}}^{\prime}\right]$ is a primitive element with respect to $N$, and denote by $\nu_{k_{0}}$ its weight. Then $N^{\nu_{k_{0}}+1}\left[\omega_{k_{0}}^{\prime}\right]=0$. For $j=0, \ldots, \nu_{k_{0}}$, put $k=k_{0}+j$. Then $\left[\omega_{k}^{\prime}\right]=\left(\frac{1}{\mu} N\right)^{j}\left[\omega_{k_{0}}^{\prime}\right]$ has order $\nu_{k_{0}}+1-j$ with respect to $N$, and weight $\nu_{k}:=\nu_{k_{0}}-2 j$. Moreover, we have $\sigma_{w}(k)=\sigma_{w}\left(k_{0}\right)+j$, as $j \mapsto s_{w}\left(k_{0}+j\right)$ is constant. The space $B_{k_{0}}:=\left\langle N^{j}\left[\omega_{k_{0}}^{\prime}\right] \mid j=0, \ldots, \nu_{k_{0}}\right\rangle$ is a Jordan block of $N$.

Assume that $k_{0} \geqslant n+1$. Then $\left[\omega_{\mu+n-k_{0}}^{\prime}\right]$ is primitive with respect to ${ }^{t} N$, hence $\left[\omega_{\mu+n-k_{0}-\nu_{k_{0}}}^{\prime}\right]$ is primitive with respect to $N$. It will be convenient to put $\bar{k}_{0}=\mu+n-k_{0}-\nu_{k_{0}}$ and, for $k=k_{0}+j$ with $j=0, \ldots, \nu_{k_{0}}, \bar{k}=\bar{k}_{0}+j$. We therefore have $\bar{k}=\mu+n-k-\nu_{k}$. Notice that, for such a $k$, we have $s_{w}(k)=$ $s_{w}\left(k_{0}\right)=\mu-s_{w}\left(\bar{k}_{0}\right)=\mu-s_{w}(\bar{k})$. We also have $\sigma_{w}(\mu+n-k)-\nu_{k}=\sigma_{w}(\bar{k})$ if $k \geqslant n+1$.

For $k \in[0, n]$, we simply put $\bar{k}=k$.

The proof of the following lemma will be given in $\S 6$.

Lemma 5.3. - For $k_{0} \geqslant n+1$, the conjugate of the Jordan block $B_{k_{0}}$ is the Jordan block $B_{\bar{k}_{0}}$, and $B_{0}$ is self-conjugate.

It follows from this lemma that, for $k$ as above, we have

$$
\overline{\left[\omega_{k}^{\prime}\right]}=\sum_{\ell=k}^{k_{0}+\nu_{0}} a_{\ell}\left[\omega_{\bar{\ell}}^{\prime}\right]
$$

with $a_{k} \neq 0$.

Let us now end the proof of Proposition 5.2. We have $F^{q} \cap W_{n+q-p}=G_{n-q} \cap M_{q-p(-1)}=\left\langle\left[\omega_{k}^{\prime}\right]\right|\left[\sigma_{w}(k)\right] \leqslant n-q$ and $\left.\nu_{k} \leqslant q-p(-1)\right\rangle$, where $(-1)$ is added if $\sigma_{w}(k) \notin \mathbb{Z}$ and not added otherwise. Therefore,

$$
\sum_{q} F^{q} \cap W_{n+q-p}=\left\langle\left[\omega_{k}^{\prime}\right] \mid\left[\sigma_{w}(k)\right]+\nu_{k} \leqslant n-p(-1)\right\rangle .
$$


Remark now that, if $k \geqslant n+1$,

$$
\begin{aligned}
{\left[\sigma_{w}(k)\right]+\nu_{k} \leqslant n-p(-1) } & \Longleftrightarrow\left[n-\sigma_{w}(\mu+n-k)\right]+\nu_{k} \leqslant n-p(-1) \\
& \Longleftrightarrow\left[\sigma_{w}\left(\mu+n-k-\nu_{k}\right)\right] \geqslant p(+1) \\
& \Longleftrightarrow\left[\sigma_{w}\left(\mu+n-k-\nu_{k}\right)\right] \geqslant p \\
& \Longleftrightarrow\left[\sigma_{w}(\bar{k})\right] \geqslant p .
\end{aligned}
$$

Arguing similarly for $k \leqslant n$, we conclude from Lemma 5.3 and (5.1) that

$$
\begin{aligned}
\sum_{q} \bar{F}^{q} \cap W_{n+q-p} & =\left\langle\overline{\left[\omega_{k}^{\prime}\right]} \mid\left[\sigma_{w}(k)\right]+\nu_{k} \leqslant n-p(-1)\right\rangle \\
& =\left\langle\left[\omega_{\bar{k}}^{\prime}\right] \mid\left[\sigma_{w}(\bar{k})\right] \geqslant p\right\rangle \\
& =H^{p} .
\end{aligned}
$$

Notice that (5.5) follows from (5.4), as $\sigma_{w}$ is increasing on each $B_{\bar{k}_{0}}$.

\section{Some topology of $f$ and proof of Lemma 5.3}

6.a. Lefschetz thimbles. - Denote by $\Delta$ the subset $\left(\mathbb{R}_{+}^{*}\right)^{n+1} \cap U \subset U$, defined by $u_{i}>0$ for $i=0, \ldots, n$. The restriction $f_{\mid \Delta}$ of $f$ to $\Delta$ takes values in $[\mu,+\infty[$ and has only one critical point (which is a Morse critical point of index 0$)$, namely $(1, \ldots, 1)$, with critical value equal to $\mu$. Notice also that $f_{\mid \Delta}$ is proper. Therefore, $\Delta$ is a Lefschetz thimble for $f$ with respect to the critical point $(1, \ldots, 1)$. Other Lefschetz thimbles at $\zeta^{\ell}(1, \ldots, 1)$ are $\zeta^{\ell} \Delta$ $(\ell=0, \ldots, \mu-1)$.

Fix $\tau \neq 0$. The morphisms

$$
H_{n}\left(U, \operatorname{Re} \tau f>C^{\prime} ; \mathbb{Q}\right) \longrightarrow H_{n}(U, \operatorname{Re} \tau f>C ; \mathbb{Q})
$$

for $C^{\prime}>C$ are isomorphisms if $C$ is big enough. We denote by $H_{n}(U, \operatorname{Re} \tau f \gg 0)$ the limit of this inverse system. This is the germ at $\tau$ of a local system $\mathcal{H}$ of rank $\mu$ on $\mathbb{C}^{*}=\{\tau \neq 0\}$. Notice that $\Delta$ defines a nonzero element of the germ $\mathcal{H}_{\tau}$ at any $\tau$ with $\operatorname{Re} \tau>0$, i.e., a section $\Delta(\tau)$ of $\mathcal{H}$ on $\{\operatorname{Re} \tau>0\}$. Therefore, it defines in a unique way a multivalued section of $\mathcal{H}$ on $\mathbb{C}^{*}$.

Let $\varepsilon>0$ be small enough. As $f$ is a $C^{\infty}$ fibration over the open set $\mathbb{C} \backslash\left\{\mu \zeta^{\ell} \mid \ell=0, \ldots, \mu-1\right\}$, it is possible to find a basis of sections $\Delta_{0}(\tau), \ldots, \Delta_{\mu-1}(\tau)$ of $\mathcal{H}$ on the open set

$$
\mathcal{S}=\left\{\tau=|\tau| e^{2 i \pi \theta} \mid \theta \in\right] \varepsilon-1, \varepsilon[\}
$$

in such a way that, for any $\ell \in\{0, \ldots, \mu-1\}$ and $|\tau|>0$, we have $\Delta_{\ell}\left(\zeta^{-\ell}|\tau|\right)=$ $\zeta^{\ell} \Delta$. Of course, this basis extends as a basis of multivalued sections of $\mathcal{H}$ on $\mathbb{C}^{*}$. 


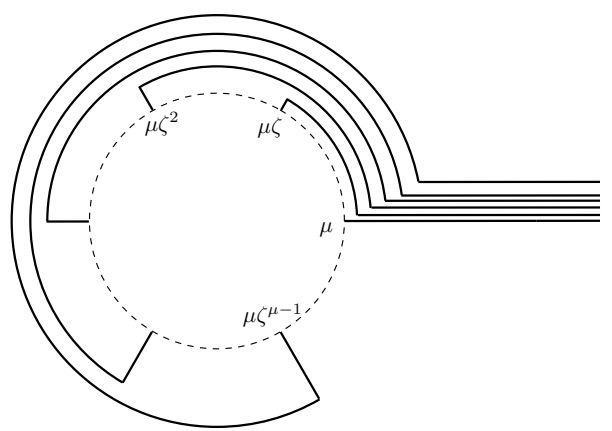

The images $f\left(\Delta_{\ell}(|\tau|)\right)$

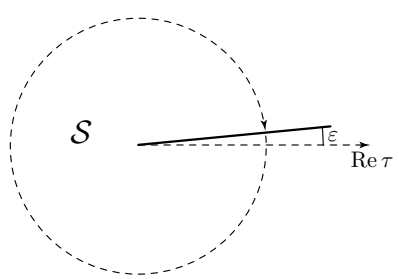

The domain $\mathcal{S}$

6.b. Integrals along Lefschetz thimbles. - Let $\eta \in G$ and let $\widetilde{\eta}$ be a representative of $\eta$ in $\Omega^{n}(U)\left[\tau, \tau^{-1}\right]$. Then the function

$$
\mathcal{S} \ni \tau \longmapsto \int_{\Delta_{\ell}(\tau)} e^{-\tau f} \widetilde{\eta}
$$

only depends on $\eta$ and is holomorphic on $\mathcal{S}$. It is denoted by $\varphi_{\ell, \eta}(\tau)$. Moreover, we have

$$
\frac{\partial \varphi_{\ell, \eta}(\tau)}{\partial \tau}=\varphi_{\ell, \partial_{\tau} \eta}(\tau)
$$

It follows that, if $\eta \in V_{\alpha} G$, then

$$
\varphi_{\ell, \eta}(\tau)=\tau^{-\alpha}\left[\sum_{m=0}^{m_{\eta}} c_{\eta, \ell}^{(m)} \frac{\left(-\frac{1}{2 i \pi} \log \tau\right)^{m}}{m !}+o(1)\right]:=\tau^{-\alpha}\left[\psi_{\eta, \ell}(\tau)+o(1)\right]
$$

where $\tau^{-\alpha}=|\tau|^{-\alpha} e^{-2 i \pi \alpha \theta}, \frac{1}{2 i \pi} \log \tau=\left(\frac{1}{2 i \pi} \log |\tau|\right)+\theta$, with $\left.\theta \in\right] \varepsilon-1, \varepsilon[$ and $c_{\eta, \ell}^{(m)} \in \mathbb{C}$. The coefficients $c_{\eta, \ell}^{(m)}$ only depend on the class $[\eta]$ of $\eta \operatorname{in~} \operatorname{gr}_{\alpha}^{V} G$, so we will denote them by $c_{[\eta], \ell}^{(m)}$, and we have

$$
c_{[\eta], \ell}^{(m)}=c_{(2 i \pi N)^{m}[\eta], \ell}^{(0)} .
$$

We will now characterize the Jordan blocks $B_{k_{0}}$ in $H_{\alpha}$. Such a Jordan block is characterized by the constant value $s$ of $s_{w}(\bullet)$, so that it will be convenient to denote such a block by $B_{\alpha, s}$.

Lemma 6.3. - For $[\eta] \in H_{\alpha}$, we have $[\eta] \in B_{\alpha, s}$ if and only if, for any $\ell=0, \ldots, \mu-1$ and any $j \geqslant 0$, we have

$$
c_{(2 i \pi N)^{j}[\eta], \ell}^{(0)}=\zeta^{\ell s} \sum_{m} c_{(2 i \pi N)^{j+m}[\eta], 0}^{(0)} \frac{(-\ell / \mu)^{m}}{m !} .
$$


Proof. - For $\eta=\omega_{k}$, denote $c_{\left[\omega_{k}\right], \ell}^{(m)}=c_{\left[\omega_{k}^{\prime}\right], \ell}^{(m)}$ by $c_{k, \ell}^{(m)}$. Then we have

$$
\int_{\Delta_{\ell}(\tau)} e^{-\tau f} \omega_{k}=\tau^{-\sigma_{w}(k)}\left(\left[c_{k, \ell}^{\left(m_{k}\right)} \frac{\left(-\frac{1}{2 i \pi} \log \tau\right)^{m_{k}}}{m_{k} !}+\cdots+c_{k, \ell}^{(0)}\right]+o(1)\right)
$$

where $m_{k}+1$ denotes the order of $\left[\omega_{k}\right]$ in $\operatorname{gr}_{\sigma_{w}(k)}^{V} G$ with respect to $2 i \pi N$. Remark now that, as $\omega_{k}=u^{a(k)} \omega_{0}$ and $|a(k)|=k$, we have

$$
\int_{\Delta_{\ell}\left(\zeta^{-\ell}|\tau|\right)} e^{-\zeta^{-\ell}|\tau| f} \omega_{k}=\int_{\zeta^{\ell} \Delta} e^{-\zeta^{-\ell}|\tau| f} \omega_{k}=\zeta^{k \ell} \int_{\Delta} e^{-|\tau| f} \omega_{k}
$$

Hence, we get

$$
\begin{aligned}
c_{k, \ell}^{\left(m_{k}\right)} \frac{\left(-\frac{1}{2 i \pi} \log |\tau|+\ell / \mu\right)^{m_{k}}}{m_{k} !} & +\cdots+c_{k, \ell}^{(0)} \\
& =\zeta^{\ell s_{w}(k)}\left[c_{k, 0}^{\left(m_{k}\right)} \frac{\left(-\frac{1}{2 i \pi} \log |\tau|\right)^{m_{k}}}{m_{k} !}+\cdots+c_{k, 0}^{(0)}\right]
\end{aligned}
$$

and in particular

$$
c_{k, \ell}^{(0)}=\zeta^{\ell s_{w}(k)} \cdot \sum_{m=0}^{m_{k}} \frac{(-\ell / \mu)^{m}}{m !} c_{k, 0}^{(m)} .
$$

Therefore, any element $[\eta]$ in $B_{\alpha, s}$ satisfies the equality of Lemma 6.3 for $j=0$, hence for any $j$.

Conversely, remark first that, if $[\eta]$ is fixed, then the equality of Lemma 6.3 for any $j \geqslant 0$ is equivalent to

$$
\psi_{[\eta], \ell}\left(\zeta^{-\ell}|\tau|\right)=\zeta^{\ell s} \psi_{[\eta], 0}(|\tau|),
$$

where $\psi_{[\eta], \ell}$ is defined by (6.1) (two polynomials are equal iff all the corresponding derivatives at 0 are equal).

Write $[\eta]=\sum \lambda_{k}\left[\omega_{k}^{\prime}\right]$ in $H_{\alpha}$, denote $m_{[\eta]}^{\prime}=\max _{k \mid \lambda_{k} \neq 0} m_{k}$ and put $K_{[\eta]}=\left\{k \mid m_{k}=m_{[\eta]}^{\prime}\right\}$. Notice that, for $k, k^{\prime} \in K_{[\eta]}$, we have $s_{w}(k) \neq s_{w}\left(k^{\prime}\right)$. If $m_{[\eta]}^{\prime}>m_{[\eta]}$, we have $\sum_{k \in K_{[\eta]}} \zeta^{\ell s_{w}(k)} \lambda_{k} c_{k, 0}^{\left(m_{k}\right)}=0$ for any $\ell=0, \ldots, \mu-1$. It follows that $\lambda_{k} c_{k, 0}^{\left(m_{k}\right)}=0$, hence $\lambda_{k}=0$, for any $k \in K_{[\eta]}$, a contradiction. Therefore $m_{[\eta]}^{\prime}=m_{[\eta]}$. Argue similarly to show that $K_{[\eta]}$ is reduced to one element, denoted by $k_{[\eta]}$, and that $s_{w}\left(k_{[\eta]}\right)=s$. Apply the lemma by induction on $m_{\left[\eta^{\prime}\right]}$ to $\left[\eta^{\prime}\right]=[\eta]-\lambda_{k_{[\eta]}}\left[\omega_{k_{[\eta]}}^{\prime}\right]$.

6.c. Isomorphism between nearby cycles. - The multivalued cycles $\Delta_{\ell}(\tau)$ form a basis of the space of multivalued global sections of $\mathcal{H}$, that we denote by $\psi_{\tau} \mathcal{H}$. This basis defines the integral (hence the real) structure on $\psi_{\tau} \mathcal{H}$. 
Denote by $\mathcal{N}_{\alpha, p}$ the space of linear combination with meromorphic coefficients of germs at $\tau=0$ of the multivalued functions $e_{\alpha, q}=\tau^{\alpha}\left(-\frac{1}{2 i \pi} \log \tau\right)^{q} / q$ ! $(q \leqslant p)$. For $p$ large enough (here $p \geqslant n+1$ is enough), the map

$$
\begin{aligned}
\varphi: V_{\alpha} G & \longrightarrow V_{0}\left(G \otimes \mathcal{N}_{\alpha, p}\right) \\
\eta & \longmapsto \sum_{j=0}^{p}\left[2 i \pi\left(\tau \partial_{\tau}+\alpha\right)\right]^{j} \eta \otimes e_{\alpha, j}
\end{aligned}
$$

induces an isomorphism

$$
\operatorname{gr}_{\alpha}^{V} G \stackrel{\sim}{\longrightarrow} \operatorname{Ker}\left[\tau \partial_{\tau}: \operatorname{gr}_{0}^{V}\left(G \otimes \mathcal{N}_{\alpha, p}\right) \longrightarrow \operatorname{gr}_{0}^{V}\left(G \otimes \mathcal{N}_{\alpha, p}\right)\right]
$$

As $G$ is regular at $\tau=0$, there exists a perturbation $\eta \mapsto \psi(\eta) \in V_{<0}\left(G \otimes \mathcal{N}_{\alpha, p}\right)$ such that $\varphi(\eta)+\psi(\eta) \in \operatorname{Ker}\left[\tau \partial_{\tau}: G \otimes \mathcal{N}_{\alpha, p} \rightarrow G \otimes \mathcal{N}_{\alpha, p}\right]$.

Recall (see, e.g., [7]) that $\operatorname{Ker}\left[\tau \partial_{\tau}: G \otimes \mathcal{N}_{\alpha, p} \rightarrow G \otimes \mathcal{N}_{\alpha, p}\right]$ is identified with $H_{\alpha}$. Set $\mathcal{N}=\oplus_{\alpha \in[0,1[} \mathcal{N}_{\alpha, n+1}$. Given a section $\lambda$ of $H=\operatorname{Ker}\left[\tau \partial_{\tau}: G \otimes \mathcal{N} \rightarrow\right.$ $G \otimes \mathcal{N}]$ and a section $\delta$ of $\psi_{\tau} \mathcal{H}$, choose a representative $\widetilde{\lambda}$ of $\lambda$ in $\Omega^{n}(U) \otimes_{\mathbb{C}} \mathcal{N}$. Then $\int_{\delta} e^{-\tau f} \widetilde{\lambda} \in \mathbb{C}$. Then (see Appendix) $\lambda$ belongs to $H_{\mathbb{Q}}$ if and only if, for any $\ell=0, \ldots, \mu$ and some nonzero $\tau$, we have

$$
\int_{\Delta_{\ell}(\tau)} e^{-\tau f} \widetilde{\lambda} \in \mathbb{Q}
$$

For $\eta \in V_{\alpha} G$ and $\widetilde{\lambda}=\varphi(\eta)+\psi(\eta)$, and using (6.1), one finds

$$
\int_{\Delta_{\ell}(\tau)} e^{-\tau f} \varphi(\eta)=c_{\eta, \ell}^{(0)}+o(1) .
$$

As a consequence, the conjugate $\overline{[\eta]}$ of $[\eta]$ satisfies

$$
c \frac{(0)}{[\eta], \ell}=\overline{c_{[\eta], \ell}^{(0)}} .
$$

It follows now from Lemma 6.3 that

$$
\overline{B_{\alpha, s}}= \begin{cases}B_{1-\alpha, \mu-s} & \text { if } \alpha \in] 0,1[, \\ B_{0, \mu-s} & \text { if } \alpha=0 .\end{cases}
$$

As $s_{w}\left(\bar{k}_{0}\right)=\mu-s_{w}\left(k_{0}\right)$, this ends the proof of Lemma 5.3.

\section{Appendix}

In this appendix, we explain with some details why the real structure on $H$ as defined by (6.6) is indeed the real structure used in [8] to define the Hodge structure on $H$. We will need to recall some notation and results of [8].

We will denote by $U$ a smooth quasi-projective variety and by $f: U \rightarrow \mathbb{A}^{1}$ a regular function on $U$. We denote by $t$ the coordinate on the affine line $\mathbb{A}^{1}$. We 
also fix an embedding $\kappa: U \hookrightarrow X$ into a smooth projective variety such that there exists an algebraic map $F: X \rightarrow \mathbb{P}^{1}$ extending $f$. We have a commutative diagram

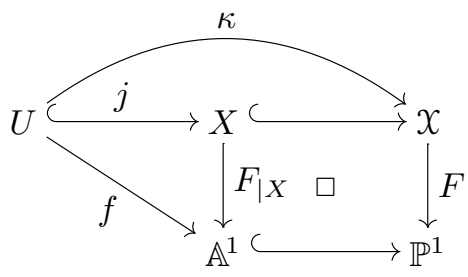

Denote by $\varepsilon: \widetilde{\mathbb{P}}^{1} \rightarrow \mathbb{P}^{1}$ the real blow-up of $\mathbb{P}^{1}$ centered at $\infty\left(\widetilde{\mathbb{P}}^{1}\right.$ is diffeomorphic to a closed disc) and by $\widetilde{F}: \widetilde{x} \rightarrow \widetilde{\mathbb{P}}^{1}$ the fibre-product of $F$ with the blowing-up $\varepsilon$. Denote by $\widetilde{\kappa}$ the inclusion $U \hookrightarrow \widetilde{X}$.

Denote by $S^{1}$ the inverse image of $\infty$ by the blowing-up $\varepsilon$. Let $\widehat{\mathbb{A}}^{1}$ be an affine line with coordinate $\tau$. Denote by $L^{\prime+}$ the closed set of $S^{1} \times \widehat{\mathbb{A}}^{1} \subset \widetilde{\mathbb{P}}^{1} \times \widehat{\mathbb{A}}^{1}$ defined by $\operatorname{Re}\left(e^{i \theta} \tau\right) \geqslant 0$, with $\theta=\arg t$ and where $t$ is the coordinate on $\mathbb{A}^{1}=\mathbb{P}^{1} \backslash\{\infty\}$, and set $L^{-}=\widetilde{\mathbb{P}}^{1} \times \widehat{\mathbb{A}}^{1} \backslash L^{\prime+}$. For $\tau \neq 0$, denote by $L_{\tau}^{\prime+}, L_{\tau}^{-} \subset \widetilde{\mathbb{P}}^{1}$ the fibre of $L^{\prime+}, L^{-}$over $\tau$.
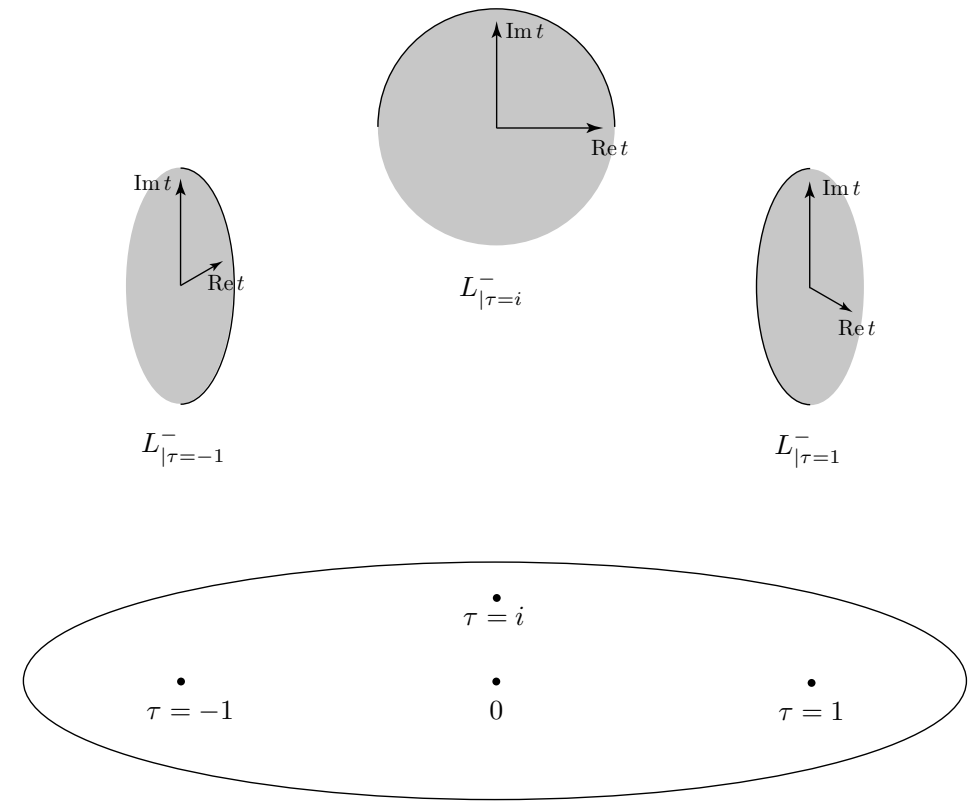

Affine line $\widehat{\mathbb{A}}^{1}$ with coordinate $\tau$ 
Denote similarly by $L_{\widetilde{x}}^{\prime+}, L_{\widetilde{x}}^{-} \subset \widetilde{x} \times \widehat{\mathbb{A}}^{1}\left(\operatorname{resp} . L_{\widetilde{x}, \tau}^{\prime+}, L_{\widetilde{x}, \tau}^{-} \subset \widetilde{x}\right)$ the inverse image of the corresponding sets by $\widetilde{F} \times \operatorname{Id}_{\widehat{\mathbb{A}}^{1}}($ resp. by $\widetilde{F})$.

We denote by $\alpha: \mathbb{A}^{1} \times \widehat{\mathbb{A}}^{1} \hookrightarrow L^{-}$and $\beta: L^{-} \hookrightarrow \widetilde{\mathbb{P}}^{1} \times \widehat{\mathbb{A}}^{1}\left(\right.$ resp. $\alpha_{\tau}: \mathbb{A}^{1} \hookrightarrow L_{\tau}^{-}$ and $\left.\beta_{\tau}: L_{\tau}^{-} \hookrightarrow \widetilde{\mathbb{P}}^{1}\right)$ the inclusions, and by the same letters the corresponding inclusions

$$
\alpha: X \times \widehat{\mathbb{A}}^{1} \hookrightarrow L_{\tilde{x}} \quad \text { and } \beta: L_{\widetilde{x}}^{-} \hookrightarrow \widetilde{x} \times \widehat{\mathbb{A}}^{1}
$$

resp.

$$
\alpha_{\tau}: X \longleftrightarrow L_{\tilde{x}, \tau}^{-} \text {and } \beta_{\tau}: L_{\tilde{x}, \tau}^{-} \longleftrightarrow \widetilde{x} .
$$

Therefore we have $\beta_{\tau} \circ \alpha_{\tau} \circ j=\widetilde{\kappa}$.

In $[8,(1.8)]$, we have defined the Fourier transform $\mathfrak{F}_{F}\left(\boldsymbol{R} j_{*} \mathbb{C}_{U}\right)$ as the following complex on $X \times \widehat{\mathbb{A}}^{1}$ (there is a shift by 1 in loc. cit., that we do not introduce here):

$$
\mathfrak{F}_{F}\left(\boldsymbol{R} j_{*} \mathbb{C}_{U}\right):=\boldsymbol{R} \varepsilon_{*} \beta_{!} \boldsymbol{R} \alpha_{*} \boldsymbol{R} j_{*} \mathbb{C}_{U \times \widehat{\mathbb{A}}^{1}},
$$

where we still denote by $j($ resp. $\kappa)$ the inclusion $U \times \widehat{\mathbb{A}}^{1} \hookrightarrow X \times \widehat{\mathbb{A}}^{1}$ (resp. $U \times$ $\widehat{\mathbb{A}}^{1} \hookrightarrow X \times \widehat{\mathbb{A}}^{1}$ ). This complex has a natural $\mathbb{Q}$-structure (replace $\mathbb{C}_{U}$ with $\left.\mathbb{Q}_{U}\right)$. This induces a $\mathbb{Q}$-structure on the nearby cycle complex $\psi_{\tau} \mathfrak{F}_{F}\left(\boldsymbol{R} j_{*} \mathbb{C}_{U}\right)=$ $\psi_{\tau} \mathfrak{F}_{F}\left(\boldsymbol{R} j_{*} \mathbb{Q}_{U}\right) \otimes_{\mathbb{Q}} \mathbb{C}$.

Denote by $\mathcal{E}^{-\tau f}$ the algebraic $\mathcal{D}_{U \times \widehat{\mathbb{A}}^{1}}$-module $\mathcal{O}_{U \times \widehat{\mathbb{A}}^{1}} e^{-\tau f}$ (i.e., the $\mathcal{O}_{U \times \widehat{\mathbb{A}}^{1}}$ module $\mathcal{O}_{U \times \widehat{\mathbb{A}}^{1}}$ with connection $\left.e^{\tau f} \circ d \circ e^{-\tau f}\right)$. The quasi-isomorphism

$$
\mathrm{DR}_{\widetilde{\mathrm{X}} \times \widehat{\mathbb{A}}^{1}}^{\mathrm{an}}\left(\kappa_{+} \mathcal{E}^{-\tau f}\right) \stackrel{\sim}{\longrightarrow} \mathfrak{F}_{F}\left(\boldsymbol{R} j_{*} \mathbb{C}_{U}\right)
$$

constructed in $[8$, Th. 2.2] is then used to define the $\mathbb{Q}$-structure on the complex (of sheaves on $X$ ) $\psi_{\tau} \mathrm{DR}_{\widetilde{X} \times \widehat{\mathbb{A}}^{1}}^{\text {an }}\left(\kappa_{+} \mathcal{E}^{-\tau f}\right.$ ) [on the other hand one uses the $V$ filtration relative to $\tau=0$ on $\kappa_{+} \mathcal{E}^{-\tau f}$ to construct the Hodge filtration on this complex]. By DR we mean the usual de Rham complex, starting in degree 0 .

Denote by $\widetilde{\mathcal{O}}_{\widetilde{\mathbb{A}}^{1}}^{\text {an }}$ the sheaf of multivalued holomorphic functions on $\widehat{\mathbb{A}}^{1} \backslash\{0\}$. Then

$$
\psi_{\tau} \mathrm{DR}_{\widetilde{\boldsymbol{X}} \times \widehat{\mathbb{A}}^{1}}^{\mathrm{an}}\left(\kappa_{+} \mathcal{E}^{-\tau f}\right)=i_{\tau=0}^{-1} \mathrm{DR}_{\widetilde{\boldsymbol{X}} \times \widehat{\mathbb{A}}^{1}}^{\mathrm{an}}\left(\kappa_{+} \mathcal{E}^{-\tau f} \otimes_{\widehat{p}^{-1} \mathcal{O}_{\widehat{\mathbb{A}}^{1}}^{\text {an }}} \widetilde{\mathcal{O}}_{\widehat{\mathbb{A}}^{1}}^{\mathrm{an}}\right),
$$

where $\widehat{p}: X \times \widehat{\mathbb{A}}^{1} \rightarrow \widehat{\mathbb{A}}^{1}$ denotes the projection and $i_{\tau=0}: \mathcal{X} \times\{0\} \hookrightarrow X \times \widehat{\mathbb{A}}^{1}$ denotes the inclusion (see, e.g., [7, (4.9.4)]). We are interested in analyzing the $\mathbb{Q}$-structure on the cohomology of $\boldsymbol{R} \Gamma\left(X, \psi_{\tau} \mathrm{DR}_{\widetilde{X} \times \widehat{\mathbb{A}}^{1}}^{\text {an }}\left(\kappa_{+} \mathcal{E}^{-\tau f}\right)\right)$. Use $C^{\infty}$ forms on $X$ to identify it with

$$
\Gamma\left(\mathcal{X}, i_{\tau=0}^{-1} \mathcal{E}_{X \times \widehat{\mathbb{A}}^{1}}^{\bullet}\left(\kappa_{+} \mathcal{E}^{-\tau f} \otimes_{\widehat{p}^{-1} \mathcal{O}_{\widehat{\mathbb{A}}^{1}}^{\text {an }}} \widetilde{\mathcal{O}}_{\widehat{\mathbb{A}}^{1}}^{\text {an }}\right)\right)
$$


with $n=\operatorname{dim} \mathcal{X}$. Similarly, denote by $\widetilde{\mathbb{C}}_{\widehat{\mathbb{A}}^{1}}$ the sheaf of multivalued local sections of $\mathbb{C}_{\widehat{\mathbb{A}}^{1}}$ (i.e., local sections on the universal covering of $\left.\widehat{\mathbb{A}}^{1} \backslash\{0\}\right)$. Then $\psi_{\tau} \mathfrak{F}_{F}\left(\boldsymbol{R} j_{*} \mathbb{C}_{U}\right)$ is equal to $i_{\tau=0}^{-1}\left(\mathfrak{F}_{F}\left(\boldsymbol{R} j_{*} \mathbb{C}_{U}\right) \otimes \widehat{p}^{-1} \widetilde{\mathbb{C}}_{\widehat{\mathbb{A}}^{1}}\right)$.

In order to know that the cohomology class of a closed multivalued section of $\widehat{p}_{*} \mathcal{E}_{\boldsymbol{X} \times \widehat{\mathbb{A}}^{1}}\left(\kappa_{+} \mathcal{E}^{-\tau f} \otimes_{\widehat{p}^{-1} \mathcal{O}_{\widehat{A}^{1}}^{\text {an }}} \widetilde{\mathcal{O}}_{\widehat{\mathbb{A}}^{1}}^{\text {an }}\right)$ is rational, one has to compute its image in $\boldsymbol{R} \widehat{p}_{*} \mathfrak{F}_{F}\left(\boldsymbol{R} j_{*} \mathbb{C}_{U}\right) \otimes \widetilde{\mathbb{C}}_{\widehat{\mathbb{A}}^{1}}$ and decide whether its class is rational or not. As the section is closed, it is enough to verify this after restricting to some (or any) $\tau \neq 0$. Therefore, we need to compute the map (A.1) after restricting to some fixed nonzero $\tau$. In (6.6), we apply this computation to the multivalued form $e^{-\tau f} \widetilde{\lambda}$.

Denote by $\mathcal{E}_{\widetilde{x}}$ the sheaf of $C^{\infty}$ functions (in the sense of Whitney) on $\widetilde{x}$, by $\mathcal{E}_{\tilde{x}}^{\text {mod }}$ the sheaf on $\widetilde{x}$ of $C^{\infty}$ functions on $U$ which have moderate growth along $\widetilde{x} \backslash U$, and by $\mathcal{E}_{\widetilde{x}}^{\bmod ,-\tau}$ the subsheaf of functions which moreover are infinitely flat along $L_{\widetilde{x}, \tau}^{\prime+}$.

On the other hand, denote by $\mathcal{C}_{U \cup L_{\tilde{x}, \tau}^{\prime+}, L_{\tilde{x}, \tau}^{\prime+}}$ the complex of sheaves on $\widetilde{x}$, consisting of germs on $\widetilde{x}$ of relative singular cochains (i.e., germs of singular cochains in $U \cup L_{\widetilde{x}, \tau}^{\prime+}$ with boundary in $L_{\widetilde{x}, \tau}^{\prime+}$ ).

By the de Rham theorem, the integration of forms induces a quasiisomorphism of complexes $\int: \mathcal{E}_{U}^{\bullet} \rightarrow \mathcal{C}_{U}^{\bullet} \otimes_{\mathbb{Z}} \mathbb{C}$; moreover, the natural morphism $\mathcal{E}_{\tilde{x}}^{\text {mod, }} \bullet \rightarrow\left(\alpha_{\tau} \circ j\right)_{*} \mathcal{E}_{U}^{\bullet}$ is a quasi-isomorphism, so the integration morphism $\int: \mathcal{E}_{\tilde{x}}^{\bmod } \bullet \rightarrow\left(\alpha_{\tau} \circ j\right)_{*} \mathcal{C}_{U}^{\bullet} \otimes_{\mathbb{Z}} \mathbb{C}$, which is obtained by composing both morphisms, is a quasi-isomorphism.

Similarly, we have a commutative diagram

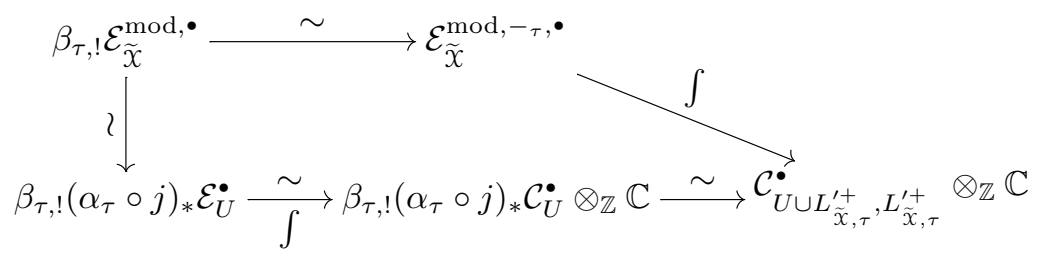

Hence we get:

Proposition A.2 (A variant of the de Rham theorem)

Both complexes $\mathcal{C}_{U \cup L_{\tilde{x}, \tau}^{\prime+}, L_{\tilde{x}, \tau}^{\prime+}}^{\prime} \otimes_{\mathbb{Z}} \mathbb{C}$ and $\mathcal{E}_{\tilde{x}}^{\text {mod, }{ }^{-\tau},} \cdot{ }^{\bullet}$ are quasi-isomorphic to $\beta_{\tau, !} \boldsymbol{R} \alpha_{\tau, *} \boldsymbol{R} j_{*} \mathbb{C}_{U}$. Moreover, the integration of forms induces a natural quasiisomorphism of complexes

$$
\int: \mathcal{E}_{\tilde{x}}^{\bmod ,-\tau}, \stackrel{\sim}{\longrightarrow} \mathcal{C}_{U \cup L_{\tilde{x}, \tau}^{\prime+}, L_{\tilde{x}, \tau}^{\prime+}}^{\bullet} \otimes_{\mathbb{Z}} \mathbb{C}
$$


Now, given a section of $\mathcal{E}_{\widetilde{x}}^{\text {mod, }} \bullet \otimes j_{+} \mathcal{E}^{-\tau f}$, i.e., a section of $\mathcal{E}_{\widetilde{x}}^{\text {mod, }} \bullet$ multiplied by $e^{-\tau f}$, it is also a section of $\mathcal{E}_{\widetilde{x}}^{\bmod ,{ }_{-\tau},}{ }^{\bullet}$, and its image by (A.1) is nothing but its integral, according to the previous commutative diagram.

\section{BIBLIOGRAPHY}

[1] S. Barannikov - "Semi-infinite Hodge structures and mirror symmetry for projective spaces", preprint, math.AG/0010157, 2000.

[2] V.V. BATYREV - "Dual polyhedra and mirror symmetry for Calabi-Yau hypersurfaces in toric varieties", J. Algebraic Geom. 3 (1994), p. 493-535.

[3] A. Douai \& C. Sabbah - "Gauss-Manin systems, Brieskorn lattices and Frobenius structures (I)", preprint, 2002.

[4] B. Dubrovin - "Geometry of 2D topological field theory", in Integrable systems and quantum groups (M. Francaviglia \& S. Greco, eds.), Lect. Notes in Math., vol. 1260, Springer-Verlag, 1996, p. 120-348.

[5] YU.I. MANin - Frobenius manifolds, quantum cohomology and moduli spaces, Colloquium Publ., vol. 47, American Mathematical Society, 1999.

[6] Z. Mebkhout - Le formalisme des six opérations de Grothendieck pour les $\mathcal{D}$-modules cohérents, Travaux en cours, vol. 35, Hermann, Paris, 1989.

[7] Z. Mebkhout \& C. SABbah - "§ III.4 D-modules et cycles évanescents", in Le formalisme des six opérations de Grothendieck pour les $\mathcal{D}$-modules cohérents [6], p. 201-239.

[8] C. SABbah - "Monodromy at infinity and Fourier transform", Publ. RIMS, Kyoto Univ. 33 (1998), p. 643-685.

[9] - "Hypergeometric period for a tame polynomial", C. R. Acad. Sci. Paris Sér. I Math. 328 (1999), p. 603-608, \& preprint math.AG/9805077.

[10] _ Déformations isomonodromiques et variétés de Frobenius, Savoirs Actuels, CNRS Éditions \& EDP Sciences, Paris, 2002.

[11] K. SAITO - "The higher residue pairings $K_{F}^{(k)}$ for a family of hypersurfaces singular points", in Singularities, Proc. of Symposia in Pure Math., vol. 40, American Mathematical Society, 1983, p. 441-463.

[12] M. Saito - "On the structure of Brieskorn lattices", Ann. Inst. Fourier (Grenoble) 39 (1989), p. 27-72. 